\title{
Mechanical performance and visual fracture warning function of mechanochromic stimuli-recovery
}

\section{polymer networks}

Yuchen Mao, Yuto Kubota, Jin Gong, Takashi Kurose, Akira Ishigami, Kota Seshimo, Takuma

Watabe, Daisuke Aoki, Hideyuki Otsuka, Hiroshi Ito*

1. Research Center for GREEN Materials \& Advanced Processing, Yamagata University, Yonezawa, Yamagata 992-8510, Japan;

2. Department of Organic Materials Science, Graduate School of Organic Materials Science, Yamagata University, Yonezawa, Yamagata 992-8510, Japan;

3. Department of Chemical Science and Engineering, Tokyo Institute of Technology, Meguroku, Tokyo 152-8550, Japan.

* $\quad$ Corresponding author: Hiroshi Ito - ihiroshi@yz.yamagata-u.ac.jp 
This file includes:

Table S1 to S2

Table S1. Mechanical properties of stimuli-recovery P(SMA-DMAA) networks in the uniaxial tensile test at room temperature

Table S2. Mechanical properties of stimuli-recovery $\mathrm{P}(\mathrm{SMA}-\mathrm{DMAA})$ networks in three loading-unloading cycles at room temperature

Figure S1 to S3

Figure S1. Dynamic modulus dissipation factor $(\tan \delta)$ as a function of the temperature of stimuli-recovery $\mathrm{P}(\mathrm{SMA}-\mathrm{DMAA})$ networks

Figure S2. DSC curves of stimuli-recovery P(SMA-DMAA) networks

Figure S3. Stress-strain curves of PN 3H (a) and PN 3 (b) in the loading-unloading cycles, and maximum stress and its recovery ratio of stimuli-recovery $\mathrm{P}(\mathrm{SMA}-\mathrm{DMAA})$ networks (c) 
Table S1. Mechanical properties of stimuli-recovery P(SMA-DMAA) networks in the uniaxial tensile test at room temperature

\begin{tabular}{|c|c|c|c|c|c|}
\hline \multirow[b]{2}{*}{ Sample } & \multicolumn{3}{|l|}{ Fracture } & \multicolumn{2}{|l|}{ Yield } \\
\hline & $\begin{array}{l}\text { Elongation } \\
(\%)\end{array}$ & $\begin{array}{l}\text { Strength } \\
\text { (MPa) }\end{array}$ & $\begin{array}{l}\text { Energy } \\
\left(\mathrm{kJ} \cdot \mathrm{m}^{-3}\right)\end{array}$ & Strain (\%) & $\begin{array}{l}\text { Stress } \\
(\mathrm{MPa})\end{array}$ \\
\hline PN 1D & $73.2 \pm 9.6$ & $3.2 \pm 0.3$ & $251.7 \pm 68.3$ & $14.0 \pm 1.5$ & $3.1 \pm 0.1$ \\
\hline PN 3D & $280.1 \pm 9.0$ & $17.0 \pm 2.1$ & $\begin{array}{l}3191.2 \\
442.8\end{array}$ & $6.3 \pm 0.4$ & $12.2 \pm 1.7$ \\
\hline PN 5D & $173.4 \pm 30.4$ & $26.3 \pm 1.4$ & $\begin{array}{l}4102.4 \\
243.8\end{array}$ & $6.4 \pm 0.2$ & $28.9 \pm 1.7$ \\
\hline PN 7D & $112.2 \pm 31.4$ & $29.0 \pm 1.4$ & $\begin{array}{l}3468.0 \\
237.6\end{array}$ & $5.3 \pm 0.5$ & $40.4 \pm 0.6$ \\
\hline $\mathrm{PN} 3 \mathrm{H}$ & $221.2 \pm 5.6$ & $15.7 \pm 0.6$ & $\begin{array}{l}2208.9 \\
123.1\end{array}$ & $7.8 \pm 0.2$ & $9.4 \pm 0.3$ \\
\hline PN 3 & $340.2 \pm 21.2$ & $7.5 \pm 1.0$ & $\begin{array}{l}2120.5 \\
118.3\end{array}$ & $9.5 \pm 1.4$ & $5.1 \pm 0.5$ \\
\hline
\end{tabular}

* Data in the table represented average value from at least two repeat tests. 
Table S2. Mechanical properties of stimuli-recovery P(SMA-DMAA) networks in three loadingunloading cycles at room temperature

\begin{tabular}{|c|c|c|c|c|c|c|}
\hline \multirow[t]{2}{*}{ Sample } & \multicolumn{2}{|l|}{$\begin{array}{l}\text { Cycle } 1 \\
\text { (original) }\end{array}$} & \multicolumn{2}{|c|}{$\begin{array}{l}\text { Cycle } 2 \\
\text { (no recovery) }\end{array}$} & \multicolumn{2}{|c|}{$\begin{array}{l}\text { Cycle } 3 \\
\text { (after heat-stimulation) }\end{array}$} \\
\hline & Stress $_{\max }{ }^{\text {a) }}$ & Energyd $^{b)}$ & StresS $_{\max }$ & EnergyD & StresS $\max$ & Energyd \\
\hline PN 3D & $14.3 \pm 0.0$ & $\begin{array}{l}2042.3 \quad \pm \\
104.4\end{array}$ & $11.8 \pm 0.1$ & $\begin{array}{l}419.9 \\
68.6\end{array}$ & $13.6 \pm 0.1$ & $\begin{array}{l}1849.7 \quad \pm \\
92.1\end{array}$ \\
\hline PN 3H & $10.7 \pm 0.4$ & $1231.5 \pm 61.6$ & $8.6 \pm 0.3$ & $\begin{array}{l}283.5 \\
38.8\end{array}$ & $9.7 \pm 0.0$ & $\begin{array}{l}1044.2 \pm \\
12.7\end{array}$ \\
\hline PN 3 & $6.4 \pm 0.1$ & $1086.2 \pm 37.4$ & $4.6 \pm 0.1$ & $\begin{array}{l}171.9 \pm \\
20.0\end{array}$ & $4.6 \pm 0.0$ & $636.8 \pm 15.1$ \\
\hline
\end{tabular}

${ }^{\text {a) }}$ Stress $\max (\mathrm{MPa})$ : Maximum stress in one loading-unloading cycle

b) Energy $\left(\mathrm{kJ} \cdot \mathrm{m}^{-3}\right)$ : Dissipated energy in one loading-unloading cycle

* Data in the table represented average value from at least two repeat tests. 


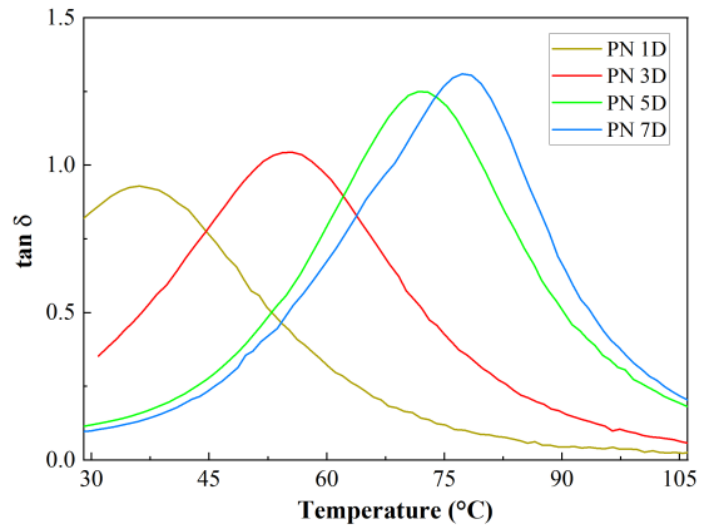

Figure S1. Dynamic modulus dissipation factor $(\tan \delta)$ as a function of the temperature of stimuli-recovery P(SMA-DMAA) networks
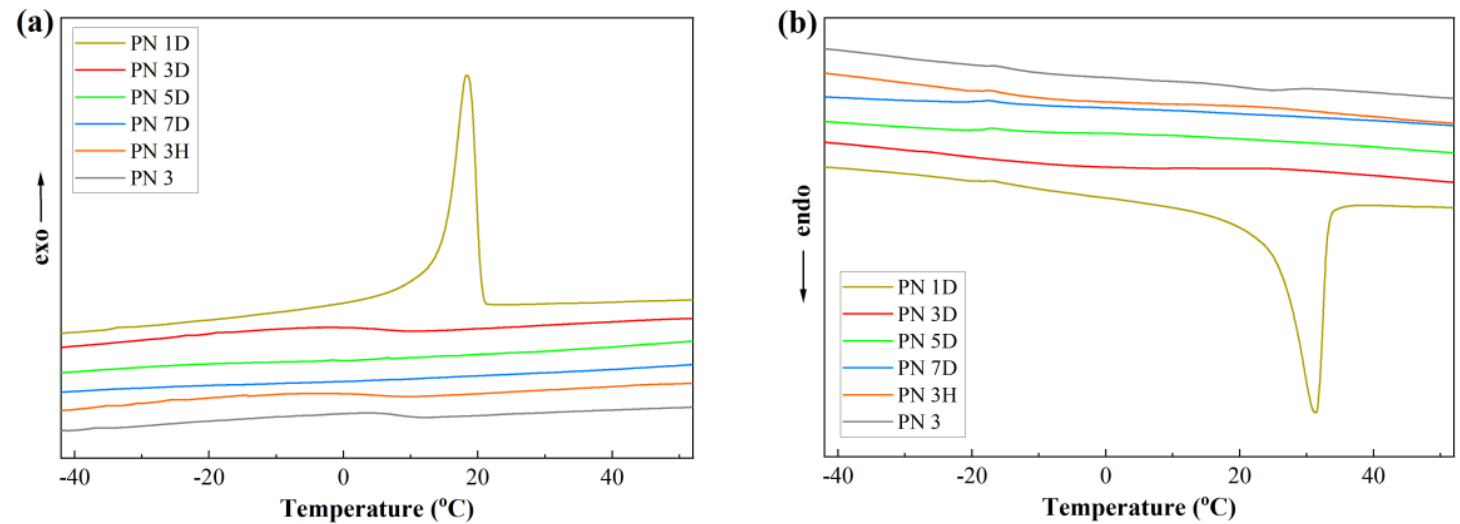

Figure S2. DSC curves of stimuli-recovery P(SMA-DMAA) networks 

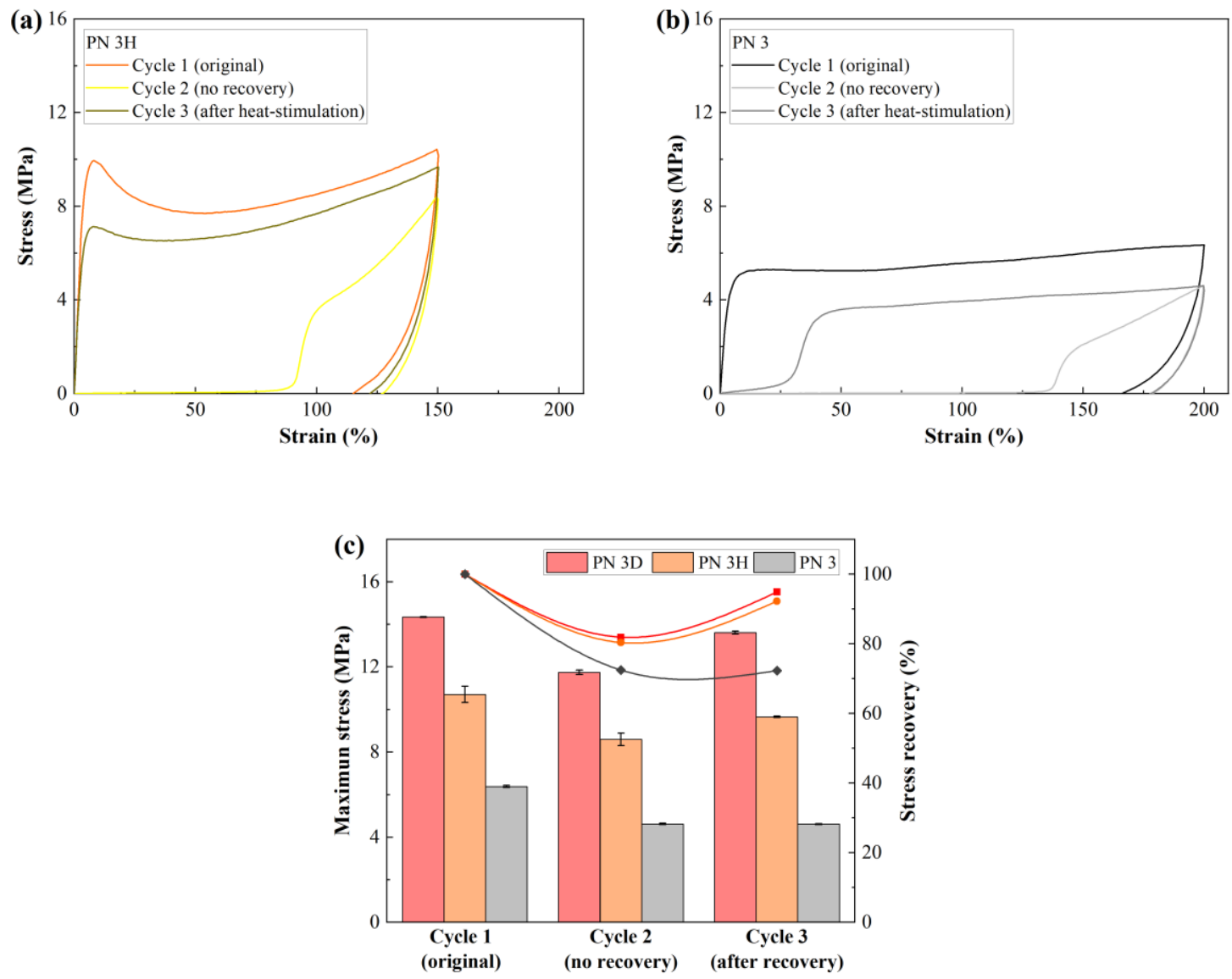

Figure S3. Stress-strain curves of PN 3H (a) and PN 3 (b) in the loading-unloading cycles, and maximum stress and its recovery ratio of stimuli-recovery P(SMA-DMAA) networks (c) 